University of Nebraska - Lincoln

DigitalCommons@University of Nebraska - Lincoln

$5-1-2000$

\title{
Forward Ion Acceleration in Thin Films Driven by a High-Intensity Laser
}

Anatoly Maksimchuk

University of Michigan, tolya@umich.edu

S. Gu

University of Michigan, Ann Arbor

K. Flippo

University of Michigan, Ann Arbor

Donald P. Umstadter

University of Nebraska-Lincoln, donald.umstadter@unl.edu

V. Yu. Bychenkov

Institute of Laser Engineering, Osaka University, Japan

Follow this and additional works at: https://digitalcommons.unl.edu/physicsumstadter

Part of the Physics Commons

Maksimchuk, Anatoly; Gu, S.; Flippo, K.; Umstadter, Donald P.; and Bychenkov, V. Yu., "Forward Ion Acceleration in Thin Films Driven by a High-Intensity Laser" (2000). Donald Umstadter Publications. 22. https://digitalcommons.unl.edu/physicsumstadter/22

This Article is brought to you for free and open access by the Research Papers in Physics and Astronomy at DigitalCommons@University of Nebraska - Lincoln. It has been accepted for inclusion in Donald Umstadter Publications by an authorized administrator of DigitalCommons@University of Nebraska - Lincoln. 


\title{
Forward Ion Acceleration in Thin Films Driven by a High-Intensity Laser
}

\author{
A. Maksimchuk,* S. Gu, K. Flippo, and D. Umstadter \\ Center for Ultrafast Optical Science, University of Michigan, \\ Ann Arbor, Michigan 48109-2099 \\ V. Yu. Bychenkov ${ }^{\dagger}$ \\ Institute of Laser Engineering, Osaka University, Suita 565-0871, Japan
}

(Received 3 September 1999)

\begin{abstract}
A collimated beam of fast protons, with energies as high as $1.5 \mathrm{MeV}$ and total number of $\gtrsim 10^{9}$, confined in a cone angle of $40^{\circ} \pm 10^{\circ}$ is observed when a high-intensity high-contrast subpicosecond laser pulse is focused onto a thin foil target. The protons, which appear to originate from impurities on the front side of the target, are accelerated over a region extending into the target and exit out the back side in a direction normal to the target surface. Acceleration field gradients $\sim 10 \mathrm{GeV} / \mathrm{cm}$ are inferred. The maximum proton energy can be explained by the charge-separation electrostatic-field acceleration due to "vacuum heating."
\end{abstract}

PACS numbers: 52.40.Nk, 52.50.Jm

Recently an interest has developed in ion acceleration by compact high-intensity subpicosecond lasers with potential applications for the initiation of nuclear reactions on a tabletop. Experiments now being carried out involve highenergy ions generated in the interaction of laser pulses with solid targets [1,2], gas jets [3,4], and clusters [5,6]. Most of the current research in this area is directed towards the development of a compact neutron source [7-10], while several other nuclear applications have recently been proposed: isotope production and initiation of fission reactions [11].

Critical for ion acceleration is the efficiency of laserenergy conversion into a high-energy electron component, since the latter through charge separation can produce the requisite strong electrostatic fields, $E$. Thermal expansion of a laser-driven plasma and ponderomotive electron expulsion constitute the most well-known examples of electrostatic-field production. While the former mechanism has been observed for many years [12], the latter one has only recently been observed in experiments with gas targets $[3,4]$. For the gas targets, when the laser pulse duration $\tau$ is long, $\tau>r_{0} / c$, where $r_{0}$ is the laser focal spot radius and $c$ is the speed of light, the radial component of the ponderomotive force dominates, and ions are accelerated radially.

For most applications, however, a collimated ion beam would be preferable. Collimation requires a planar charge separation, which can be achieved by focusing an intense laser onto the surface of a planar solid-density film. In this case laser light terminates at the target surface and drives high-energy electrons generated in front of it deep inside the target. Because of planar charge separation these electrons produce a strong electrostatic field accelerating ions in a forward direction.

In this Letter, we report on the direct observation of high-energy ion beam accelerated by this means [13]. Similar experiments simultaneously were performed in two other laboratories, namely in the Rutherford Appleton Laboratory on a Vulcan laser and in the Lawrence Livermore National Laboratory on a Petawatt laser [14,15]. In our experiments we observed that the protons, which appear to originate from impurities on the front side of the thin-film target, are accelerated over a region extending into the target and exit out the back side, in a direction normal to the target surface. The results are found to depend on a plasma scale length and for a high laser-contrast case can be explained by the "vacuum heating" mechanism at a sharp interface due to the Brunel effect [16] or the $\mathbf{v} \times \mathbf{B}$ Lorentz force [17]. In previously reported experimental studies [1], the accelerated ions were found to propagate in the direction of plasma expansion, and the acceleration was attributed to charge displacement by thermal expansion.

The experiments were carried out with 10 TW hybrid Ti:sapphire/Nd:phosphate glass chirped pulse amplification laser, which is able to deliver up to $4 \mathrm{~J}$ in $400 \mathrm{fs}$ pulse at the wavelength of $1.053 \mu \mathrm{m}$ with the intensity contrast ratio of $\sim 10^{4}: 1$, as measured by the third order correlation technique. The contrast has been improved to an estimated $10^{7}: 1$ by the frequency doubling of laser light in a $4-\mathrm{mm}$ KDP crystal. In these experiments the energy in a green light was limited to $1 \mathrm{~J}$, because of the nonlinear distortion of the laser wave front in a doubling crystal. The laser beam was focused on the surface of thin films of aluminum with a thickness of $1.8 \mu \mathrm{m}$ at $0^{\circ}$ or $45^{\circ}$ incidence angle with an $f / 3(f=16.5 \mathrm{~cm})$ off-axis parabolic mirror. The maximum focused intensity was $3 \times 10^{18} \mathrm{~W} / \mathrm{cm}^{2}$ for $2 \omega_{0}$.

The high-energy ion emission was recorded by CR-39 plastic nuclear track detector, which is able to record ions with energies $\geq 100 \mathrm{keV} /$ nucleon as tracks on a surface of the detector, after being etched in sodium hydroxide solution. To study the angular ion distribution we have used CR-39 covered with single thickness Mylar filters ranging from 2 to $26 \mu \mathrm{m}$. To determine a maximum energy 
and spectrum of the ions, the detectors were covered with steps of Mylar filters with thicknesses from 2 to $50 \mu \mathrm{m}$. To compare ion emission in the forward (through the thin-film target) and backward directions (the direction of plasma expansion) for $45^{\circ}$ incidence angle, two CR-39 detectors, with sets of Mylar filters, were placed both in front and in back of the target.

It was found that the predominant component of highenergy ion emission are protons. These protons originate from a thin layer on the target surface contaminated with hydrocarbon and/or water vapor. Such target contamination was observed in the late 1960s in laser-matter interaction experiments with nanosecond laser pulses as discussed in Ref. [12] and has been proven in the 1990s in experiments with subpicosecond high-intensity pulses $[1,2]$. In our experiments, we observed a high-energy proton beam, propagating inside a thin-film target and emerging through the rear surface as a beam with the angular divergence of about $40^{\circ} \pm 10^{\circ}$ (Fig. 1a). The proton angular distribution is peaked in the center of the beam, where the maximum density of ion tracks was observed (Fig. 1a). The direction of the high-energy proton beam does not depend on the angle of incidence of laser radiation and is always normal to the target surface. From this fact one would conclude that the electric field of charge separation accelerating protons inside the target is directed along the target surface normal.

To verify that most of the energetic ions are indeed protons, we used a dipole magnetic spectrometer with a

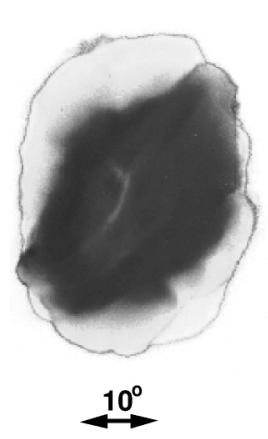

(a)

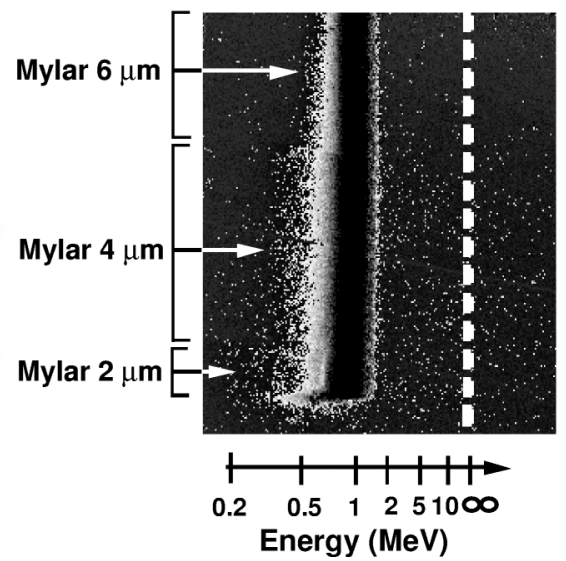

(b)
FIG. 1. (a) A typical image of a proton beam observed in the forward (behind the target) direction in the interaction of $1.8-\mu \mathrm{m}$-thick aluminum foil with a high-intensity laser. The proton beam passed through a $25 \mu \mathrm{m}$ Mylar filter, which corresponds to an energy above $1.2 \mathrm{MeV}$. The laser intensity on target is $2 \times 10^{18} \mathrm{~W} / \mathrm{cm}^{2}$ at $2 \omega_{0}$ illumination normal to the target. (b) Spectrogram of fast protons emitted in the forward direction and deflected in a dipole magnetic spectrometer. A dashed line shows the position of the slit image without the magnet. CR-39 was used as a detector and covered with three steps of Mylar filter of 2,4 , and $6 \mu \mathrm{m}$ thickness, which corresponds to proton cutoff energies of $0.2,0.3$, and $0.5 \mathrm{MeV}$.
200- $\mu \mathrm{m}$ slit in front of the magnet. CR-39 plastic with three steps of Mylar film in front was used as a detector. The Mylar filters had thicknesses of 2, 4, and $6 \mu \mathrm{m}$, which correspond to proton cutoff energies of $0.2,0.3$, and $0.5 \mathrm{MeV}$, respectively. Figure $1 \mathrm{~b}$ presents a spectrogram of high-energy ions obtained with the dipole magnet. It shows a very sharp cutoff for particle energies of about $1.5 \mathrm{MeV}$, which was consistent with results on maximum proton energies as will be discussed below. On the left side of the spectrogram (Fig. 1b) by the arrows we have shown positions of above-mentioned cutoff energies. These positions agree within $\pm 10 \%$ of accuracy to the deflection of protons in the magnetic field, expressed by the formula: $x \simeq L Z e b B / \sqrt{2 \epsilon_{i} M}$, where $L$ is the distance between magnet and detector, $Z=1$ for proton, $e$ is the electron charge, $b$ is the width of the magnet, $B$ is the magnetic field strength, and $\epsilon_{i}$ and $M$ are the ion energy and mass.

We study the dependence of maximum proton energy versus laser intensity for different conditions of $\mathrm{Al}$ thin foil illumination. The highest proton energy of $1.6 \mathrm{MeV}$ has been observed for normal incidence at the maximum laser intensity of $3 \times 10^{18} \mathrm{~W} / \mathrm{cm}^{2}$ (Fig. 2a). For $45^{\circ}$ angle of incidence the highest intensity on a target was reduced by a factor of 2 due to increased spot size in the horizontal direction. Maximum observed proton energy, $\epsilon_{i}^{\max }$, was comparable for both cases for the same intensity and can be fitted as a function $\epsilon_{i}^{\max } \propto I^{\alpha}$, where $\alpha$ is between 0.3 and 0.4. Illumination with $s$ polarization at $45^{\circ}$ has produced protons with energy $200-300 \mathrm{keV}$ less than for $p$ polarization. We also observed high-energy protons in the backward direction (in the direction of a plasma expansion) for $45^{\circ}$ laser illumination, but their energy was twice lower than those in the forward direction. We changed the plasma gradient scale length by introducing a prepulse at $2 \omega_{0}$, with the time delay of $50 \mathrm{ps}$ in front of the main $2 \omega_{0}$ pulse. We varied the prepulse intensity from $0.01 \%$ to $10 \%$ of main pulse intensity of $10^{18} \mathrm{~W} / \mathrm{cm}^{2}$. We found that there is an optimum in prepulse intensity of about

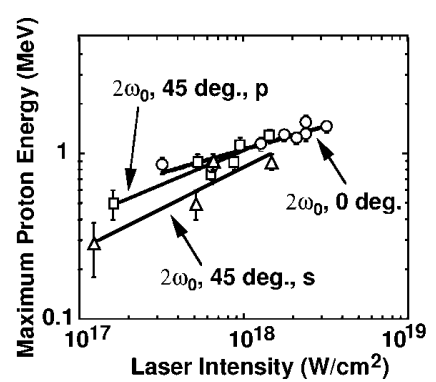

(a)

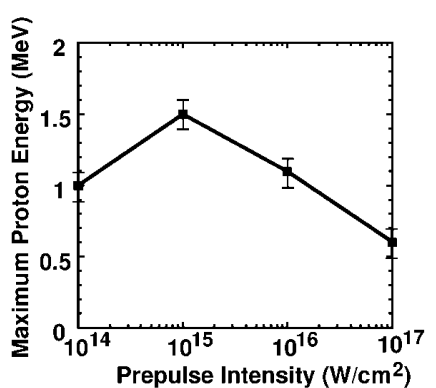

(b)
FIG. 2. The maximum proton energy in the forward direction as a function of (a) laser intensity at $2 \omega_{0}$ and for different conditions of illumination of $1.8-\mu \mathrm{m} \mathrm{Al}$ foil: circles $-0^{\circ}$; squares $-45^{\circ}, p$ - polarization; triangles - $45^{\circ}, s$ - polarization. Solid lines are the best power function fits to the experimental data. (b) The $2 \omega_{0}$ prepulse intensity at $10^{18} \mathrm{~W} / \mathrm{cm}^{2}$ main pulse intensity. 
$10^{15} \mathrm{~W} / \mathrm{cm}^{2}$ (Fig. 2b) for maximum proton energy production. We estimated that at this intensity the scale length of the preformed plasma is about a few laser wavelength.

The proton energy spectrum was measured by the foil attenuation method. Use of steps of Mylar filters, with thicknesses differing by $2 \mu \mathrm{m}$ provided proton energy resolution of about $100 \mathrm{keV}$. The proton spectrum (Fig. 3a) shows an exponential decay from energies of $400 \mathrm{keV}$ to $1 \mathrm{MeV}$ with a characteristic temperature of $230 \mathrm{keV}$ (solid line). An interesting feature of the spectrum at higher energy is a plateau, which ends in a sharp energy cutoff at $1.5 \mathrm{MeV}$ that is typical for the electrostatic mechanism of ion acceleration [11]. To prove that high-energy ions are mainly accelerated from the front side of the target, which was in contradiction with [15] we performed an experiment on activation of the sample of boron isotope ${ }^{10} \mathrm{~B}$ with deuterons [18]. The sample was placed behind the target at the distance of about $1 \mathrm{~cm}$. In the first set of experiments we deposited a thin layer of a deuterated plastic on a front side of a $6 \mu \mathrm{m}$ thick Mylar foil. In this case we observed approximately $10^{5}$ atoms of positron active isotope ${ }^{11} \mathrm{C}$ produced by the reaction ${ }^{10} \mathrm{~B}(d, n){ }^{11} \mathrm{C}$. In the second set of experiments, when a thin layer of a deuterated plastic was on a back side of a Mylar foil, no activation has been measured. These results indicate unequivocally that for the condition of our experiment ions are accelerated from the front side of the thin foil. To determine the scale length of ion acceleration we varied the thickness of aluminum film from 0.1 to $25 \mu \mathrm{m}$. Circles on Fig. $3 \mathrm{~b}$ are the experimental data points showing the dependence of maximum proton energy versus thickness $l_{0}$ of the film. For the thinnest target with $l_{0}=0.1 \mu \mathrm{m}, \epsilon_{i}^{\max }$ was about $1 \mathrm{MeV}$. We observed an increase in the maximum detected proton energy versus target thickness for $l_{0}<10 \mu \mathrm{m}$. It reaches a peak at

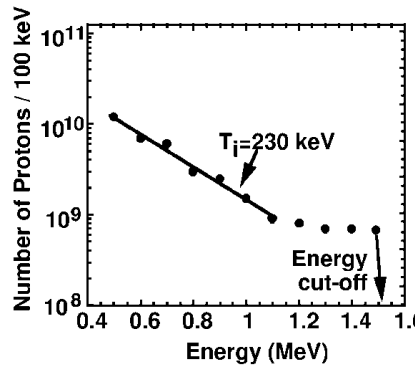

(a)

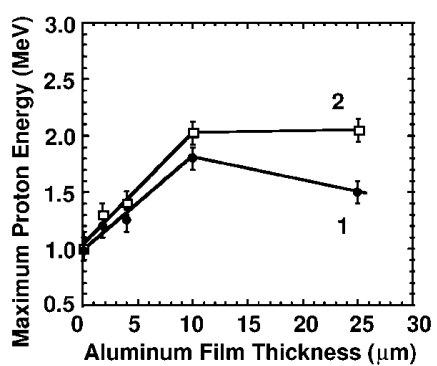

(b)
FIG. 3. (a) Energy distribution of fast protons in the forward direction (circles), measured by attenuation of the beam in Mylar filters of different thicknesses for $2 \omega_{0}$ illumination of $1.8-\mu \mathrm{m} \mathrm{Al}$ film with intensity of $2 \times 10^{18} \mathrm{~W} / \mathrm{cm}^{2}$ at normal incidence. An arrow shows a sharp cutoff in proton energy on a spectral distribution below a detection threshold. The detection threshold was about $10^{6}$ protons $/ 100 \mathrm{keV}$. (b) Maximum energy of protons for laser intensity of $1.5 \times 10^{18} \mathrm{~W} / \mathrm{cm}^{2}$ at $2 \omega_{0}$ and $0^{\circ}$ angle of incidence as a function of aluminum foil thickness - experiment (squares); ion losses are excluded (circles). $l_{0} \simeq 10 \mu \mathrm{m}$. At larger thicknesses the proton energy decreases. This experiment demonstrates very efficient ion acceleration at a short spatial scale at the target front side where the predominant ion acceleration occurs and where ions gain their characteristic energy. For the maximum laser intensity we also have studied the dependence of maximum proton energy as a function of atomic number $Z$ for $2 \mu \mathrm{m}$ thick carbon, Mylar, molybdenum, and lead foils. We did not observe a significant difference in the maximum proton energies for different materials.

In spite of the absence of self-consistent theory of highenergy electron and ion generation in the laser-solid target interactions, we may propose a heuristic pragmatic approach to get qualitative estimations. We will consider an interaction of a high-contrast laser pulse with an intensity $I>10^{18} \mathrm{~W} / \mathrm{cm}^{2}$ at normal incidence in which the high-energy electrons with relativistic velocities $v \sim$ $c$ can be produced. We assume when a high-intensity high-contrast laser pulse terminates at a target surface it produces a plasma with a size $l \approx \lambda / 2$ [19], due to the longitudinal electron oscillations resulting from $\mathbf{v} \times \mathbf{B}$ oscillating Lorentz force. Near the target-vacuum surface the electrons are pushed in and out by the oscillating component of the ponderomotive force. Inside the target this force sharply vanishes. Twice in a laser period electrons reenter the target. Returning electrons are accelerated by the "vacuum" electric field and then deposit their energy inside the target. The electrons of this plasma are strongly heated by laser light, penetrate inside the solid target with relativistic velocities, and constitute a low density $\left(n_{e}<n_{c}\right)$ high-energy component of the entire electron population. They acquire an energy $\epsilon_{e}$ from the laser pulse which can be evaluated from energy balance: $n_{e} \epsilon_{e} c \approx \eta I$, where $n_{e}$ is the electron density of small size preformed plasma and coefficient $\eta$ defines the efficiency of laser-energy conversion into the high-energy electrons. For laser intensities in the range of $10^{17}-3 \times 10^{18} \mathrm{~W} / \mathrm{cm}^{2}$ we estimate $\eta$ in a range from a few percent to $15 \%$, in accordance with previous measurements [20] in similar experimental conditions.

The mean free path of high-energy electrons is very large-a $1 \mathrm{MeV}$ free electron penetrates solid aluminum over a distance of $\sim 1.5 \mathrm{~mm}$. Nevertheless, in a plasma it is limited to a Debye length $\lambda_{D e}$ due to the charge-separation field, which for an electron density $n_{e} \sim 10^{19}-10^{20} \mathrm{~cm}^{-3}$ is comparable with the laser wavelength. High-energy electrons penetrating into a solid target (or even passing through it for the very thin foils) create an electrostatic field with $e \phi \approx \epsilon_{e}$, or $E \sim \epsilon_{e} / e \lambda_{D e} \sim 10 \mathrm{GeV} / \mathrm{cm}$, which accelerates ions in the forward direction, and in turn decelerate. An electrostatic field near the target surface has a bipolar structure with the more pronounced component accelerating ions in a forward direction. If the laser pulse duration is larger than the ion acceleration time in the layer of size $\lambda_{D e}$, as is the case of our experiment, ions acquire an electrostatic energy $\epsilon_{i} \approx Z e \phi \approx Z \epsilon_{e}$. 
It has been known that the nonlinear regime of vacuum heating provides accelerated electrons with energies higher than predicted by the $\mathbf{v} \times \mathbf{B}$ force or $P$ component of the laser field. This is related to the strong "heating" due to the self-intersection of electron orbits [21]. Instead of returning to the target in each cycle, many electrons remain on the front of a target and form a timeaveraged density profile. In order to blow the electrons from the preformed plasma layer, the laser intensity must be enough to accelerate electrons up to an energy exceeding the Coulomb energy [22]: $\epsilon_{e} \gtrsim 2 \pi e^{2} n_{e} l R$, where $R$ is the radius of focal spot, $R \gg l(R \simeq 5 \mu \mathrm{m})$. From this relation and energy balance, one can estimate the characteristic electron density $n_{e} \lesssim n_{c}(a / 2 \pi) \sqrt{\eta 2 \lambda / R}$ and energy $\epsilon_{e} \geqslant \pi a m c^{2} \sqrt{\eta R / 2 \lambda}$, where $n_{c}$ is the critical density and $a=0.85 \times 10^{-9} \lambda[\mu \mathrm{m}] \sqrt{I\left[\mathrm{~W} / \mathrm{cm}^{2}\right]}$ is the normalized amplitude of laser-field vector potential. Consequently, the maximum ion energy can be evaluated as $\epsilon_{i}^{\max } \gtrsim Z \sqrt{\eta I R \lambda}$, where the laser intensity and spatial scales are measured in units of $10^{18} \mathrm{~W} / \mathrm{cm}^{2}$ and microns, respectively. For laser intensity of $3 \times 10^{18} \mathrm{~W} / \mathrm{cm}^{2}$ and for $\eta=10 \%, \epsilon_{i}^{\max } \gtrsim 1 \mathrm{MeV}$, that is, in agreement with the experimental data (Fig. 2a). We may compare our estimate with an estimate based on a ponderomotive potential [23], which has been used for the description of ion acceleration in gas targets [3,11] $\epsilon_{i} \approx Z m c^{2}\left[\sqrt{1+a^{2}}-1\right]$. This formula predicts an ion energy an order of magnitude lower than observed in the present experiment at an intensity of $3 \times 10^{18} \mathrm{~W} / \mathrm{cm}^{2}$. It is evident also that the thermal expansion of a skin-layer plasma cannot explain $\mathrm{MeV}$ ion generation because of low bulk electron temperature there. One may believe that stochastic electron heating due to parametric processes in the plasma corona can provide the high temperature component of electrons, $T_{h}$, and corresponding high thermal expansion velocity $\sim \sqrt{T_{h} / M}$, where $M$ is the ion mass. However, at $2 \omega_{0}$ illumination with a high laser-intensity contrast the micron spatial scale of a plasma corona prevents such processes.

We may explain also why ion energy cutoff depends on the foil thickness (Fig. 3b, curve 1). As follows from the estimations, the typical $\lambda_{D e}$ is about $1 \mu \mathrm{m}$ so that for the foil thickness $l_{0} \lesssim 1 \mu \mathrm{m}$ electrons will penetrate to the back side. Even for thicker targets ultrafast electrons $(>1 \mathrm{MeV})$, which cannot be stopped by charge separation, leave the target. Nevertheless, for the thick foils all electrons stop in the target and participate in generation of the electric field accelerating the ions. Because of this, one may expect an increase in the maximum ion energy with the foil thickness unless saturation occurs. However, ion energy losses increase with the foil thickness that should result in the optimum thickness, which according to Fig. $3 \mathrm{~b}$, curve 1 , corresponds to $l_{0} \sim 10 \mu \mathrm{m}$. To demonstrate the effect of the saturation mentioned we plot also the similar dependence, $\epsilon_{i}^{\max }\left(l_{0}\right)$, where ion energy losses are excluded (Fig. 3b, curve 2).
In summary, we have demonstrated the production of a directed beam of high-energy protons in the interaction of high-intensity laser with a thin-film target. We have shown that the generation of fast ions occurs on the front side of the target and the maximum proton energy agrees with an estimation of charge separation due to hot-electron generation by the vacuum heating effect. Such a beam of energetic protons might serve as a high-current proton injector or be used in basic science to study nuclear transformations on a picosecond time scale. A charge compensated high-energy deuteron beam can also be used as a fast ion ignitor for the direct and indirect drive inertial confinement fusion research.

A. M., K. F., and the laser facilities were supported by the National Science Foundation. S.G. and D. U. were supported by the Division of Chemical Sciences, Office of Basic Energy Sciences, U.S. Department of Energy. V. Yu. B. is thankful for the hospitality of the Institute of Laser Engineering of the Osaka University. The work of V. Yu. B. was supported in part by the Russian Foundation for Basic Research (Grant No. 00-02-16063). The authors thank K. Nemoto and S. Banerjee for providing the data on boron activation with deuterons, G. Mourou and Y. Y Lau for useful discussions, and the staff of Michigan Ion Beam Laboratory for help in the calibration of CR-39 detectors.

*Electronic address: tolya@umich.edu

${ }^{\dagger}$ On leave from P. N. Lebedev Physics Institute, Moscow 117924, Russia.

[1] A. P. Fews et al., Phys. Rev. Lett. 73, 1801 (1994); F. N. Beg et al., Phys. Plasmas 4, 447 (1997).

[2] P. E. Young et al., Phys. Rev. Lett. 76, 3128 (1996).

[3] G. S. Sarkisov et al., JETP Lett. 66, 828 (1997); G. S. Sarkisov et al., Phys. Rev. E 59, 7042 (1999).

[4] K. Krushelnick et al., Phys. Rev. Lett. 83, 737 (1999).

[5] T. Ditmire et al., Nature (London) 386, 54 (1997).

[6] M. Lezius et al., Phys. Rev. Lett. 80, 261 (1998).

[7] P. A. Norreys et al., Plasma Phys. Controlled Fusion 40, 175 (1998).

[8] G. Pretzler et al., Phys. Rev. E 58, 1165 (1998).

[9] T. Ditmire et al., Nature (London) 398, 489 (1999).

[10] L. Disdier et al., Phys. Rev. Lett. 82, 1454 (1999).

[11] V. Yu. Bychenkov et al., Sov. Phys. JETP 88, 1137 (1999).

[12] S. J. Gitomer et al., Phys. Fluids 29, 2679 (1986).

[13] A. Maksimchuk et al., Bull. Am. Phys. Soc. 44, 229 (1999).

[14] E. Clark et al., Bull. Am. Phys. Soc. 44, 182 (1999).

[15] R. D. Snavely et al., Bull. Am. Phys. Soc. 44, 228 (1999).

[16] F. Brunel, Phys. Rev. Lett. 59, 52 (1987).

[17] W. L. Kruer and K. Estabrook, Phys. Fluids 28, 430 (1985).

[18] K. Nemoto et al. (to be published).

[19] W. Yu et al., Phys. Rev. E 58, 2456 (1998).

[20] J. Yu et al., Phys. Plasmas 6, 1318 (1999).

[21] S. V. Bulanov et al., Phys. Plasmas 1, 745 (1994).

[22] T.Zh. Esirkerov et al., JETP Lett. 70, 82 (1999).

[23] S. C. Wilks, Phys. Fluids B 5, 2603 (1993). 